NUV femtosecond laser inscription of volume Bragg gratings in poly(methyl)methacrylate with linear and circular polarizations

This content has been downloaded from IOPscience. Please scroll down to see the full text.

2013 Laser Phys. 23126004

(http://iopscience.iop.org/1555-6611/23/12/126004)

View the table of contents for this issue, or go to the journal homepage for more

Download details:

IP Address: 138.253.100.121

This content was downloaded on 27/01/2014 at 14:00

Please note that terms and conditions apply. 


\title{
NUV femtosecond laser inscription of volume Bragg gratings in poly(methyl)methacrylate with linear and circular polarizations
}

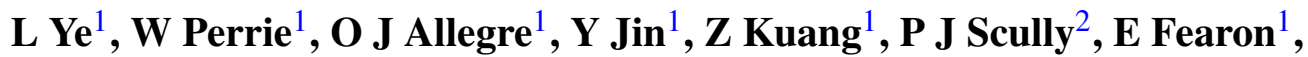 \\ D Eckford ${ }^{1}$, S P Edwardson ${ }^{1}$ and G Dearden ${ }^{1}$ \\ ${ }^{1}$ Laser Group, School of Engineering, University of Liverpool, L69 3GQ, UK \\ 2 The Photon Science Institute, The University of Manchester, M13 9PL, UK \\ E-mail: wpfemto1@liv.ac.uk
}

Received 20 August 2013

Accepted for publication 25 October 2013

Published 19 November 2013

Online at stacks.iop.org/LP/23/126004

\begin{abstract}
Large, high efficiency, volume Bragg gratings with dimensions of $5 \mathrm{~mm} \times 5 \mathrm{~mm}$ and thickness between 1 and $7 \mathrm{~mm}$ with $20 \mu \mathrm{m}$ pitch have been inscribed in poly(methyl)methacrylate (PMMA) with $180 \mathrm{fs}, 387 \mathrm{~nm}$ parallel beams using both linear and circular polarizations. Linear polarization (perpendicular to the scan direction) produced the highest refractive index contrast, while circular polarization produced the lowest. The measured first-order diffraction efficiency with grating thickness $L$ agrees well with theoretical expectations, and reached a maximum of $94 \%$ near $L=4 \mathrm{~mm}$, the highest yet observed in pure PMMA. The source of the variation in refractive index contrast was investigated, and it was found to be due to the polarization-dependent nonlinear filamentation, the first such observation in a pure polymer.
\end{abstract}

(Some figures may appear in colour only in the online journal)

\section{Introduction}

Volume Bragg gratings (VBGs) are used in a wide range of interesting applications, such as astronomical spectroscopy, ultrafast laser compressors and wavelength division multiplexing [1], wavelength stabilization of high power laser diodes [2], and narrowband filters for Raman spectroscopy [3]. Further, chirped VBGs have been used in the stretching and compression of ultrafast laser pulses in fibre laser chirped pulse amplification (CPA) systems, eliminating the dispersion mismatch of the stretcher/compressor [4]. VBGs are generally produced holographically in photosensitive materials such as dichromated gelatine (DCG) films typically $10-30 \mu \mathrm{m}$ thick with refractive index (RI) modulation $\Delta n \sim 0.02-0.1$ [5] or photothermorefractive (PTR) glass, where modified thickness can be several millimetres with $\Delta n \sim 0.001$ [6]. Such gratings are remarkably stable, and in the case of PTR material can handle high average laser powers exceeding $100 \mathrm{~W}$ with damage thresholds $F \sim$ $10 \mathrm{~J} \mathrm{~cm}^{-2}$ [7]. Careful design can result in devices with high diffraction efficiency close to unity combined with line densities $>1000$ lines $\mathrm{mm}^{-1}$.

Femtosecond laser inscription, initiated by multi-photon absorption, has extended the field of microstructured optical materials to almost all transparent dielectrics, including glasses [8-13] and polymers [14-25]. Thanks to their ease of manufacture and low cost, polymers are attractive substrates for the fabrication of photonic components such as transient optical memory [14], photonic crystals [15], 2D and 3D waveguides [16, 17], 3D microchannels [18], splitters [19], and VBGs [20]. In the latter case, holographic exposure of azodye-doped PMMA with NIR, 150 fs pulses yielded $0.3 \mathrm{~mm}$ diameter VBGs with a first-order Bragg diffraction efficiency (DE) $\eta_{1} \sim 90 \%$ and 200 lines $\mathrm{mm}^{-1}$. However, inscription 


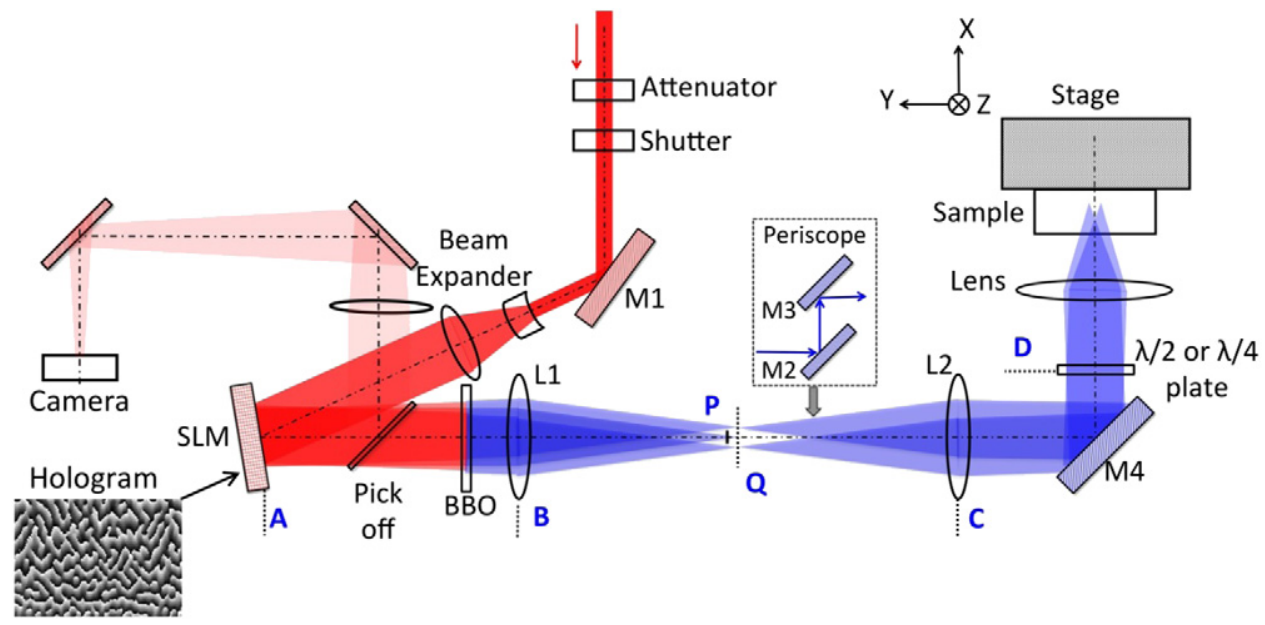

Figure 1. Experimental set-up. The CGH applied to a phase-only SLM creates multiply diffracted NIR beams which are frequency doubled in the BBO crystal.

simultaneously created surface relief gratings, contributing an estimated $\sim 8 \%$ to this high efficiency.

Pure PMMA is of interest because it has high optical transparency, and is inexpensive, and so is ideal for creating disposable photonic devices for clinical, biological, and chemical applications. For example, NIR, low NA femtosecond laser inscription of $200 \mu \mathrm{m}$ thick VBGs in undoped PMMA was first demonstrated using $40 \mathrm{fs}, 1 \mathrm{kHz}$ pulses at $800 \mathrm{~nm}$ with DE $\eta_{1} \sim 37 \%$ and inferred $\Delta n \sim$ $5 \times 10^{-4}$ [21]. Mochizuki et al [22] produced $10 \mu \mathrm{m}$ pitch, $300 \mu \mathrm{m}$ thick VBGs in PMMA and other polymers, using $0.5 \mu \mathrm{J}, 120 \mathrm{fs}$ pulses at $800 \mathrm{~nm}$ and $1 \mathrm{kHz}$ repetition rate, focused with a low NA (0.13) objective, with highest $\eta_{1} \sim 45 \%$ in poly(methyl)pentene. More efficient inscription in PMMA at $800 \mathrm{~nm}$ occurs with temporal pulselengths $\tau_{\mathrm{p}} \leq 100 \mathrm{fs}$, allowing overscanning of exposed regions, thus increasing $\Delta n$ without optical breakdown [23]. At low NA $(\sim 0.1)$, filamentation occurs in dielectrics, leading to a dynamic balance between Kerr lens focusing and plasma defocusing, extending modification depths well beyond the Rayleigh length, ideal for creating thick VBGs [24]. By frequency doubling longer, $160 \mathrm{fs}$ NIR pulses at $775 \mathrm{~nm}$ to the NUV, excellent inscription in PMMA was achieved at $387 \mathrm{~nm}$, and the photochemistry mechanisms were investigated after exposure [25].

Single-beam inscription, especially at low $\mathrm{kHz}$ repetition rates, can be accelerated by the use of parallel processing using a spatial light modulator (SLM), capable of generating arbitrary spot patterns when addressed with appropriate computer-generated holograms (CGHs) [26]. Using 16 parallel NIR beams with $775 \mathrm{~nm}, 160$ fs pulses at $1 \mathrm{kHz}$, high uniformity VBGs were inscribed in PMMA, reaching $\eta_{1}=70 \%$ with $4 \mathrm{~mm}$ thickness and inferred $\Delta n \sim 4.6 \times$ $10^{-5}$ [27]. By combining an SLM with a BBO nonlinear crystal, parallel beam NUV inscription was used to fabricate $2 \mathrm{~mm}$ thick VBGs reaching $\eta_{1}>70 \%$ and hence inferred $\Delta n \sim 8.4 \times 10^{-5}$ [28]. The use of $387 \mathrm{~nm}, 180 \mathrm{fs}$ pulses in PMMA allows nonlinear modification via 2-photon ionization rather than 3-photon ionization, required at $775 \mathrm{~nm}$, resulting in higher $\Delta n$ and more stable gratings [27, 28].

In this study, the parallel beam technique developed by us previously [28] is used to inscribe a series of large, high quality VBGs with thickness ranging from $L=1$ to $7 \mathrm{~mm}$ in clinical grade PMMA with $180 \mathrm{fs}, 387 \mathrm{~nm}$ parallel beams at low NA $(\sim 0.1)$. The measured first-order diffraction efficiency when inscribed with linear vertical polarization $|V\rangle$ (transverse electric, TE) reached $\eta_{1}>94 \%$ near $L=$ $4 \mathrm{~mm}$, the highest yet observed in pure PMMA, fitting the expected theoretical function. With $L>4 \mathrm{~mm}$, the phase angle $\phi>\pi / 2$, and hence coupling back to zero order is observed. Further, by altering the incident state of polarization during inscription, a polarization-dependent refractive index modulation was detected in PMMA with maximum $\Delta n$ measured with linear (TE) polarization. This is the first observation of polarization-dependent filamentation in a pure polymer as far as the authors are aware.

\section{Experimental details}

The experimental set-up, figure 1, has been described previously in detail [28], and so only a brief description is given for completeness. The main difference is the use of a phase-only SLM for this work. The horizontally polarized output beam from a Clark-MXR CPA 2010 system $(775 \mathrm{~nm}$, $160 \mathrm{fs}, 1 \mathrm{kHz}, 1 \mathrm{~mJ}$ ) is attenuated then expanded on to a phase-only SLM (Hamamatsu X-10468-02, 800 pixels $\times$ 600 pixels) addressed with appropriate $\mathrm{CGH}$ which generates multiply diffracted NIR beams. A thin BBO crystal $8 \mathrm{~mm} \times$ $8 \mathrm{~mm} \times 0.7 \mathrm{~mm}, \theta=30^{\circ}, \phi=0^{\circ}$ is placed after and in close proximity to the SLM, converting the NIR beams to nearly collinear NUV beams. A $4 \mathrm{f}$ optical system with two plano-convex lenses (L1, L2, $\mathrm{f}=194 \mathrm{~mm}$ at $\lambda=$ $387 \mathrm{~nm}$ ) is set to reimage the SLM surface to the input plane of a fused silica objective lens (f.l. $=50 \mathrm{~mm}$, NA $\sim 0.1)$. The undiffracted zero-order NIR and NUV components were blocked by a small target near the Fourier plane of the first lens L1. The PMMA substrates 


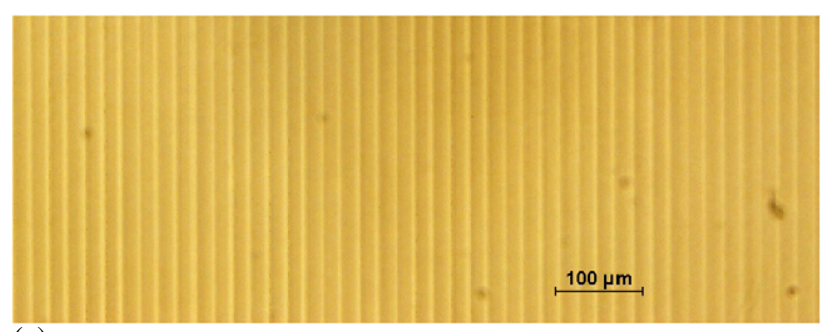

(a)

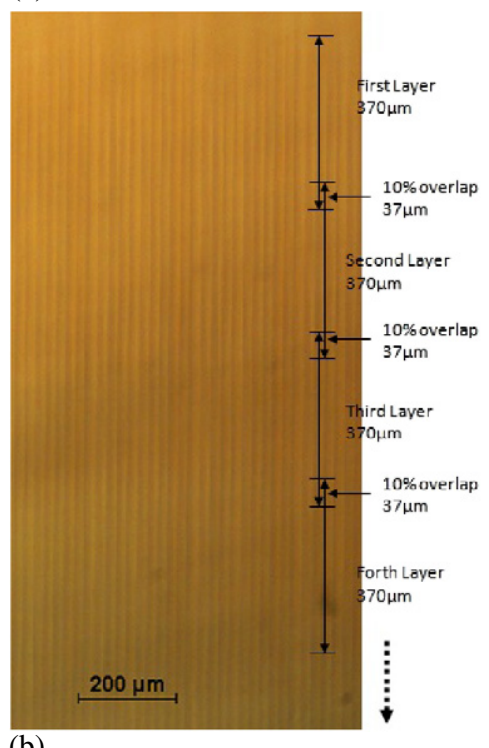

(b)

Figure 2. (a) Microscope image of front view of continuous filaments and (b) $1.5 \mathrm{~mm}$ thick cross section of VBG in PMMA.

(Vistacryl CQ non-UV, $25 \mathrm{~mm} \times 25 \mathrm{~mm} \times 7.5 \mathrm{~mm}$ ), polished on all sides, were mounted on a precision 3 -axis motion control system (Aerotech) running under NView MMI and the $387 \mathrm{~nm}$ parallel beams were carefully focused $>100 \mu \mathrm{m}$ below the surface. Addition of a zero-order half-wave/quarter-wave plate at objective input plane D allowed polarization-dependent inscription.

\section{Results-vertical linear polarization $|\mathrm{V}\rangle$ (transverse electric, TE)}

We define the incident polarization state with the ket notation, $|\mathbf{P}\rangle$. Phase hologram CGHs were generated in a LabView environment with the iterative Gerchberg-Saxton algorithm [29], generating 10 near uniform, paraxial vertically diffracted spots, frequency doubled in BBO to $\lambda_{2 \omega} \sim 387 \mathrm{~nm}$, creating vertically linearly polarized beams. A series of VBGs with thickness from 1 to $7 \mathrm{~mm}$ with pitch $\Lambda_{1}=22.3 \mu \mathrm{m}$ was inscribed horizontally at constant pulse energy and vertical linear polarization $|V\rangle$ transverse to the scan direction. Filaments were observed to have length $\delta L=373 \pm 28 \mu \mathrm{m}$ and width $W \sim 4.0 \mu \mathrm{m}$ when inscribed with pulse energy $E_{\mathrm{p}}=0.31 \mu \mathrm{J} /$ beam at $0.08 \mathrm{NA}$ and $s=1 \mathrm{~mm} \mathrm{~s}^{-1}$ scan speed. The pulse energies were set carefully and monitored using a pyroelectric detector, measuring every pulse at $1 \mathrm{kHz}$ (type JE-05, Molectron). A continuously modified cross section was

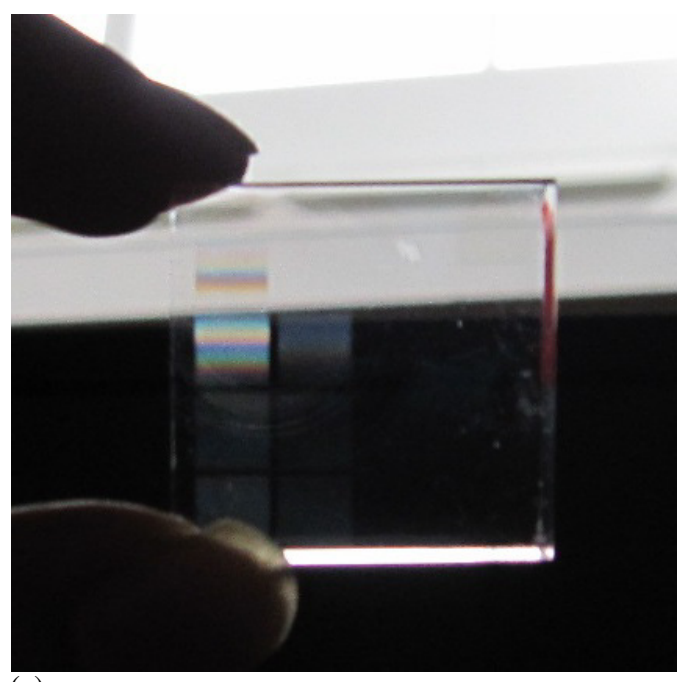

(a)

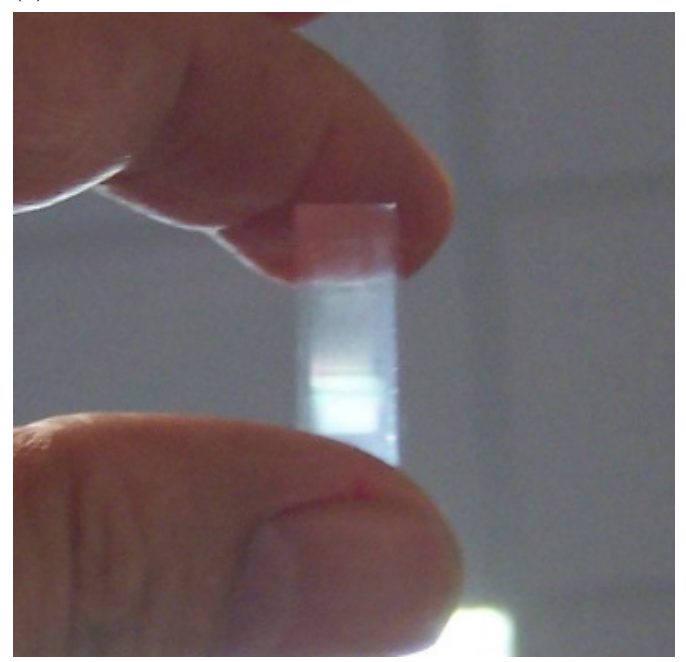

(b)

Figure 3. (a) Photo of $5 \times 5 \times[1-7] \mathrm{mm}^{3}$ volume phase gratings. (b) Side view of thick VBGs in PMMA.

created by stitching filaments separated by $\Delta L=333 \mu \mathrm{m}$ along the optic axis, hence with a $10 \%$ overlap, previously shown to be satisfactory [28], while offsetting the comb of spots at a pitch $\Lambda_{2}=10 \Lambda_{1}$ in the vertical, to extend the $3 \mathrm{D}$ grating size. Figure 2(a) shows a front view of a VBG with pitch $\Lambda_{1}=22.3 \mu \mathrm{m}$, while figure 2(b) shows a continuous VBG cross section of thickness $t>1.5 \mathrm{~mm}$ with four filament overlaps.

Figure 3(a) shows a photo of $5 \times 5 \times[1-7] \mathrm{mm}^{3}$ volume phase gratings in PMMA, made at the speed of $9 \mathrm{~min} \mathrm{~mm}^{-1}$ thickness ( $3 \mathrm{~min} /$ layer, 3 layers $\mathrm{mm}^{-1}$ ), while figure $3(\mathrm{~b})$ shows a side view of thick VBG structures.

Figures 4(a)-(c) show the diffraction of the transmitted beam at $\lambda=532 \mathrm{~nm}$ with 2 and $5 \mathrm{~mm}$ thick VBGs. The spots were viewed on a distant wall $\sim 1.2 \mathrm{~m}$ away. The samples were mounted carefully on a mirror mount and rotation stage so that the gratings could be brought to normal incidence prior to diffraction measurements. The $2 \mathrm{~mm}$ thick VBG also shows a faint coupling to -1 st order at the Bragg angle, figure 4(b) while the $5 \mathrm{~mm}$ grating shows high first-order coupling with 


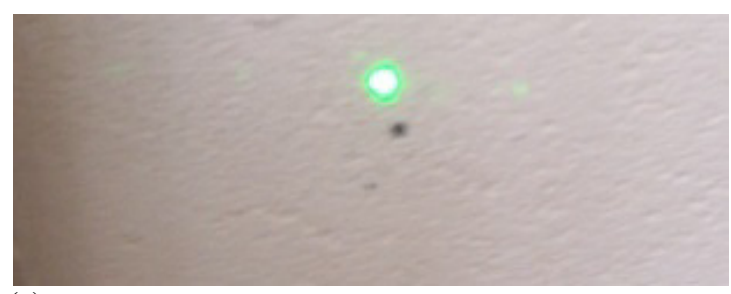

(a)

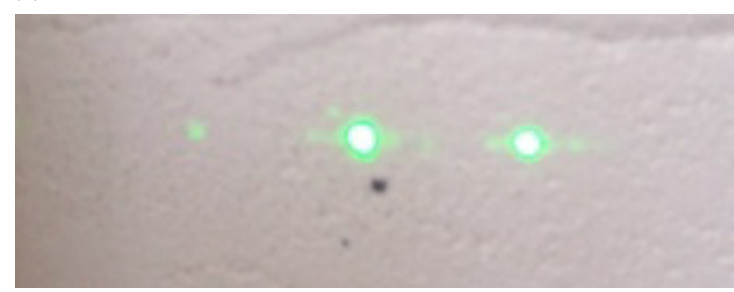

(b)

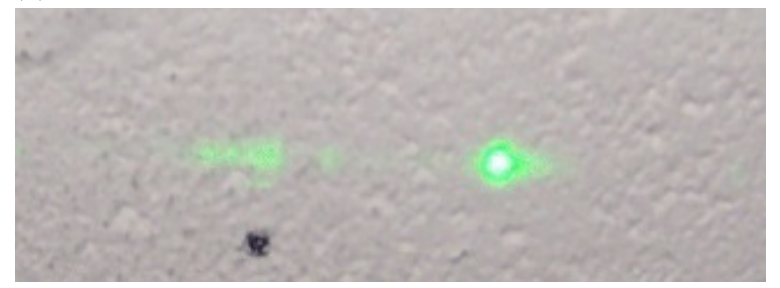

(c)

Figure 4. First-order diffraction of VGBs. (a) $L=2 \mathrm{~mm}$, normal incidence, zero order, near dot, (b) $L=2 \mathrm{~mm}$, grating rotated to first order Bragg angle showing DE $\eta_{1} \sim 40 \%$, (c) $L=5 \mathrm{~mm}$ thick VBG, first-order Bragg diffraction with $\eta_{1}>90 \%$.

$\eta_{1}>90 \%$. Zero order appears just above the black dot. There is clearly remaining background scatter, mainly near the zero order.

The fabrication time for a $5 \mathrm{~mm} \times 5 \mathrm{~mm} \times 5 \mathrm{~mm}$ thick VBG was $\sim 45$ min with ten parallel spots, scanned in only one direction to avoid potential non-uniformity effects due to pulse front tilt [30], present in most femtosecond laser compressors. As the gratings develop after inscription [28] and $\Delta n$ increases, the first-order efficiency alters accordingly. Figure 5 summarizes the measured first-order DE of the seven gratings over 30 days after inscription since the gratings continue to develop over this period before stabilizing. Gratings with thickness $L=1-4 \mathrm{~mm}$ have DE that increases monotonically with time as $\Delta n$ increases. However, the gratings with $L=5-7 \mathrm{~mm}$ reach a maximum DE within hours to days, and then decay afterwards. The grating with $L=7 \mathrm{~mm}$ reached its highest diffraction efficiency almost immediately after inscription (within hours) and decreased thereafter as the modulation $\Delta n$ increased.

From the data of figure 5, figure 6(a) shows the diffraction efficiency measured at the Bragg angle a few hours and 30 days after inscription, and comparison with the theoretical fit due to Kogelnik [31], $\eta_{ \pm 1}=$ $\sin ^{2} \phi=\sin ^{2}\left(\pi \Delta n L / \lambda \cos \theta_{\mathrm{B}}\right)$, where $\Delta n \sim \Delta n(t)$ is the RI modulation, $L$ is the grating thickness, $\lambda=532 \mathrm{~nm}$ is the read out wavelength, and $\theta_{\mathrm{B}}$ is the Bragg angle. The fits to the Kogelnik expression are pleasing, supporting a near uniform modification throughout the structures, and they highlight the developing RI modulation, thus pushing the diffraction

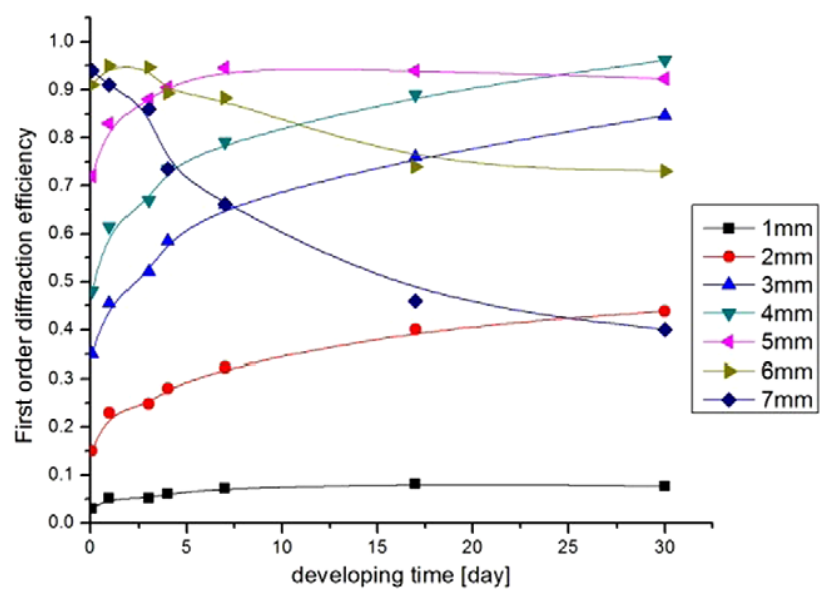

Figure 5. Measured VBG first-order DE with time for seven gratings with thickness $L=1-7 \mathrm{~mm}$ and pitch $\Lambda=22.3 \mu \mathrm{m}$. The curves are guides to the eye.

maxima where $\phi>\pi / 2$ to lower $L$. After 30 days, the RI modulation $\Delta n^{\text {lin }}=(6.01 \pm 0.07) \times 10^{-5}$ and the inferred optimum grating thickness is $L \sim 4.5 \mathrm{~mm}$. At higher $L$, the phase angle $\phi>\pi / 2$, so the efficiency drops as light couples back to zero order in these interferometric structures. The RI modulation $\Delta n^{\text {lin }}$ is relatively low but not unexpected with single transverse scans of the waveguides with $180 \mathrm{fs}$ pulses. The low NA used (0.08) allows deep uniform modification (up to $10 \mathrm{~mm}$ ), as spherical aberration at the interface is essentially negligible [32].

The Bragg angular envelope of a $5 \mathrm{~mm}$ thick grating, measured at $532 \mathrm{~nm}, 17$ days after inscription is shown in figure 6(b) and compared with a theoretical fit [33],

$$
\eta(\Delta \theta)=\sin ^{2} \frac{\left[\pi L\left(\frac{\Delta n}{\lambda}\right)^{2}+\left(\frac{\Delta \theta}{\Lambda}\right)^{2}\right]^{0.5}}{\left[1+\left(\frac{\lambda \Delta \theta}{\Lambda \Delta n}\right)^{2}\right]},
$$

where $L$ is the grating thickness, $\Delta n=\Delta n^{\text {lin }}$ is the $\mathrm{RI}$ modulation, $\Delta \theta$ is the angular deviation from the Bragg angle $\theta_{\mathrm{B}}$, and $\Lambda$ is the grating pitch. The measured $\Delta \theta_{\text {fwhm }}^{\exp } \sim$ $1.3 \Delta \theta_{\mathrm{fwhm}}^{\text {theory }}$, so the envelope is marginally broader than expected, most likely due to the slight angular divergence of the filaments which have a low but finite deviation [28]. The efficiency peaks near $\theta_{\mathrm{B}}=\sin ^{-1}\left(\frac{\lambda}{2 \Lambda}\right)=0.68^{\circ}$, as expected. The angular response also confirms that the grating is indeed a thick volume grating with thickness parameter [31] $Q=$ $\frac{2 \pi \lambda L}{n \Lambda^{2}}=18$ with $L=4 . Q>10$ is required for thick gratings. Using this definition, the gratings for $L \geq 2 \mathrm{~mm}$ are all thick. VBGs were also found to be remarkably insensitive to reading wavelength polarization, one of the attributes of VBGs.

\subsection{Inscription effects with linear and circular polarizations}

A series of $5 \mathrm{~mm}$ gratings with pitch $\Lambda_{1}=22.3 \mu \mathrm{m}$ was inscribed under identical conditions except that the incident state of polarization was either linear vertical $|V\rangle$ (perpendicular to scan direction, TE), linear horizontal $|H\rangle$ (parallel to scan direction, TM), or circular $(|R\rangle$ and $|L\rangle$ ). The 


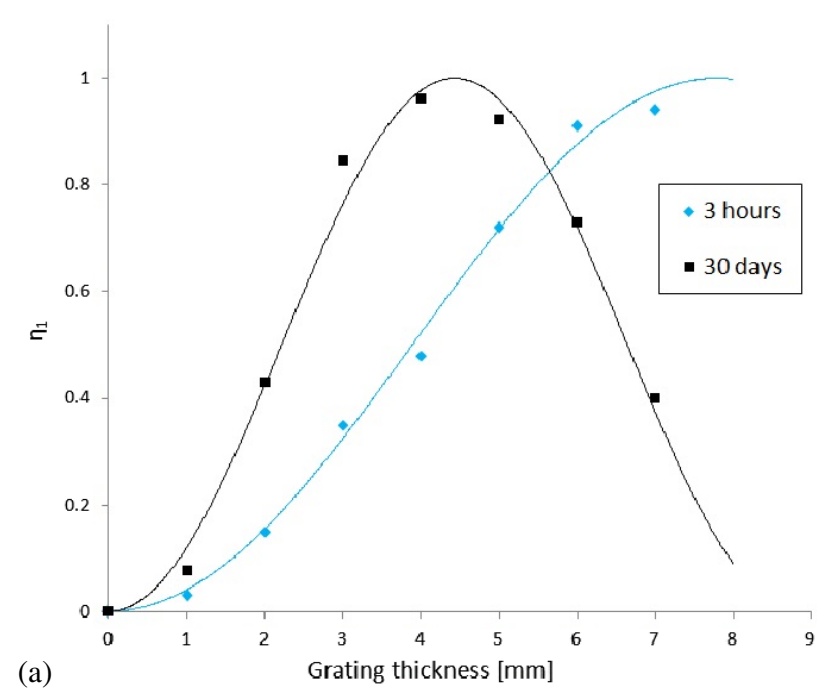

(a)

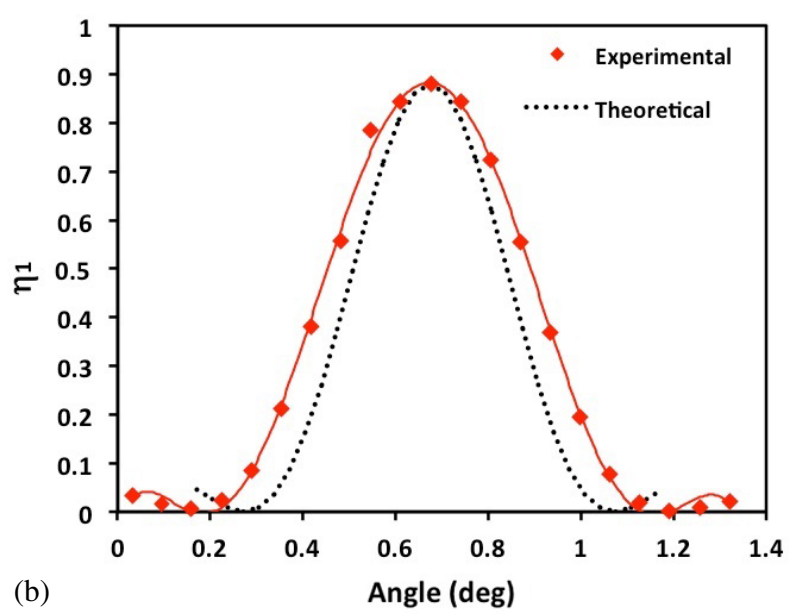

Figure 6. (a) Top: measured first-order DE of seven gratings, a few hours and 30 days after inscription and comparison with theory (solid lines) $\eta_{1}=\sin ^{2}(\phi)$, where $\Delta n=3.1 \times 10^{-5}$ and $6.0 \times 10^{-5}$ respectively. (b) Bottom: measured Bragg angular envelope at $532 \mathrm{~nm}$ of a $4 \mathrm{~mm}$ thick, $\Lambda=22.3 \mu \mathrm{m}$ pitch $\mathrm{VBG}$ and comparison with theoretical fit [33].

polarization was altered using either a zero-order half-wave $(|V\rangle,|H\rangle)$ or quarter-wave $(|R\rangle,|L\rangle)$ plate, inserted just ahead of the objective. A clear polarization-dependent inscription was observed. First-order DE was highest with vertical polarization $|V\rangle$ (TE), then linear horizontal $|H\rangle$ (TM), and finally circular polarization $(|R\rangle$ and $|L\rangle)$. As expected from symmetry, the first-order diffraction efficiency $\eta_{1}(|R\rangle)=$ $\eta_{1}(|L\rangle)$ within experimental error.

Table 1 summarizes the measured first-order Bragg efficiencies, nine days after inscription. Linear polarization states clearly couple more strongly to the material. Errors indicate $1 \sigma$. While $\eta_{1}(|V\rangle)-\eta_{1}(|H\rangle) \sim 5 \%$, the highest difference $\eta_{1}(|V\rangle)-\eta_{1}(|R\rangle) \sim 8 \%$, corresponding to $\delta(\Delta n)=$ $6 \%$ and $9 \%$ respectively. The total pulse energy was monitored carefully throughout the inscription of the sets of four VBGs and maximum variation in pulse energy $\frac{\Delta E_{\mathrm{p}}}{E_{\mathrm{p}}}=$ $\frac{0.02 \mu \mathrm{J}}{3.12 \mu \mathrm{J}}=0.64 \%$, where $E_{\mathrm{p}}$ is the total pulse energy for ten spots.

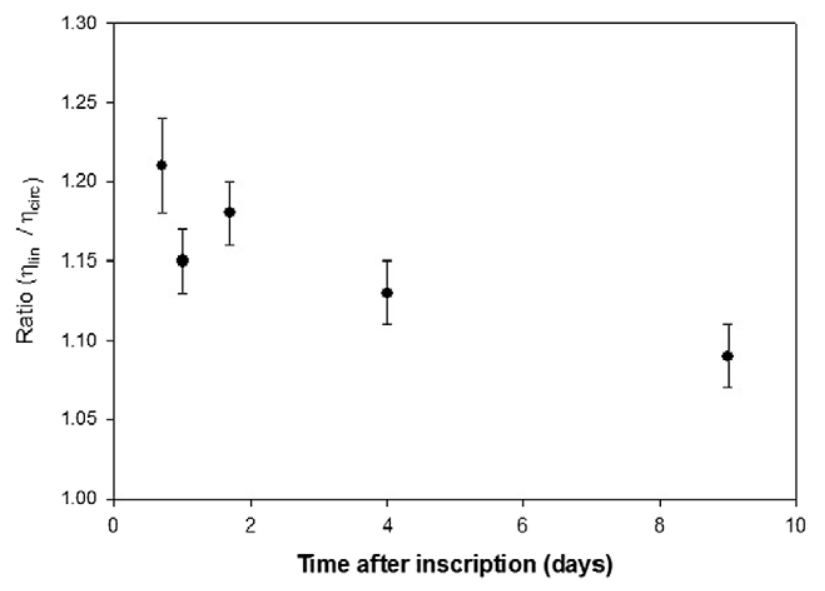

Figure 7. Measured ratio of first-order diffraction efficiencies $\eta_{1}(|V\rangle) / \eta_{1}(|R\rangle)$ with time.

Table 1. First-order diffraction efficiencies nine days after inscription with different polarizations.

\begin{tabular}{lll}
\hline Polarization & $\eta_{1}(L=5 \mathrm{~mm})$ & Inferred $\Delta n$ \\
\hline$|V\rangle$ & $0.92 \pm 0.02$ & $(4.35 \pm 0.13) \times 10^{-5}$ \\
$|H\rangle$ & $0.87 \pm 0.02$ & $(4.07 \pm 0.10) \times 10^{-5}$ \\
$|R\rangle$ & $0.84 \pm 0.02$ & $(3.92 \pm 0.10) \times 10^{-5}$ \\
$|L\rangle$ & $0.85 \pm 0.02$ & $(3.97 \pm 0.10) \times 10^{-5}$ \\
\hline
\end{tabular}

The measured ratio $\eta_{1}(|V\rangle) / \eta_{1}(|R\rangle)$ was time dependent; see figure 7. Thus, $\left[\eta_{1}(|V\rangle) / \eta_{1}(|R\rangle)\right]_{\max }=1.21 \pm 0.03,(1 \sigma)$ shortly after inscription. This ratio decays to $1.09 \pm 0.02$ after nine days, as the gratings develop.

To investigate the effects of polarization inscription during filamentation, the filament widths and lengths were investigated after exposure. Figure 8 shows optical images of parallel filaments written with vertical linear (TE) and circular polarizations. The filament widths, measured over sets of ten, were found to be essentially independent of the writing polarization and the average width over many filaments and all polarizations, $\langle W\rangle=4.02 \pm 0.02 \mu \mathrm{m}(1 \sigma)$.

Sets of filaments were inscribed using single-beam inscription with linear (TE) and circular polarizations with a pulse energy $E_{\mathrm{P}}=0.301 \pm 0.0013 \mu \mathrm{J}(1 \sigma)$. The detector response (J3-05, Molectron) was checked carefully and determined to be independent of polarization within experimental error. For these experiments, the quarter-wave plate fast axis orientation was rotated from vertical, parallel to the incident linear polarization $|V\rangle$, to $\pm 45^{\circ}$ to generate $|R\rangle$ and $|L\rangle$ circular polarizations. Figures 9(a) and (b) show the optical images (in transmission) of the filamentary modification cross sections observed between filaments inscribed with linear (TE) and circular polarizations. There is clearly a significant difference in filamentary modifications. With linear polarization, the inscribed filaments appear more uniform, and appear to have higher RI contrast than with circular polarization $\left(\Delta n^{\text {lin }}>\Delta n^{\text {circ }}\right.$ ), while also originating nearer the laser source by around $\Delta L \sim 40 \mu \mathrm{m}$. As expected from symmetry, right and left polarizations generate similar 


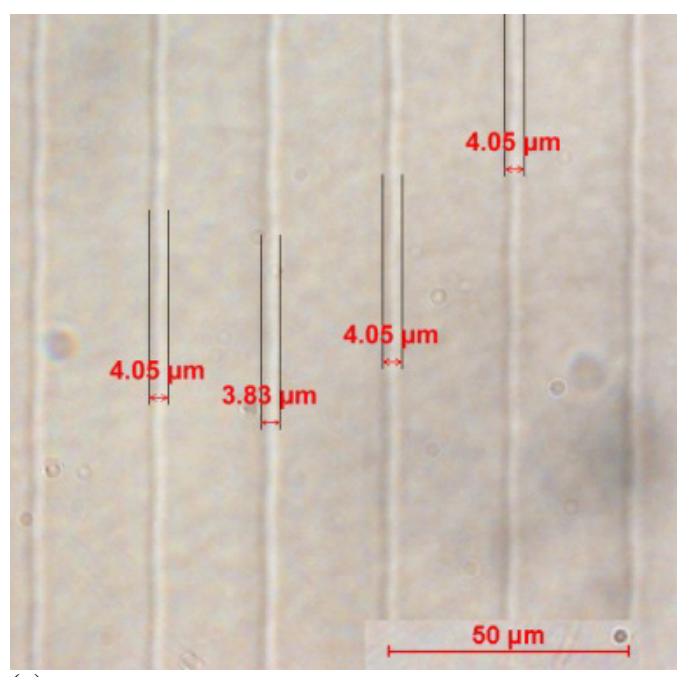

(a)

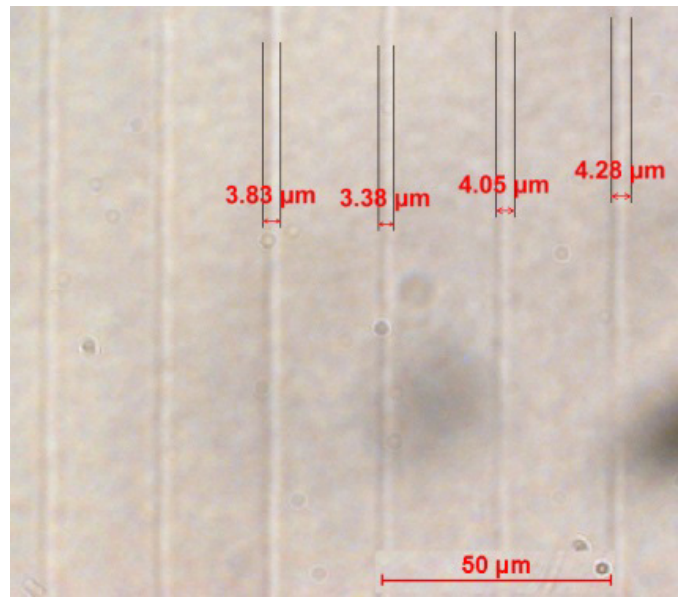

(b)

Figure 8. Widths of parallel beam filament cross sections for vertical (TE) (a) and circular (b) polarizations.

modifications; see figure 9(b), which shows filament cross sections observed at a depth of $\sim 2 \mathrm{~mm}$. However, circular polarization also shows a short, higher contrast region $\left(\Delta n^{\text {circ }}>\Delta n^{\text {lin }}\right.$ near $\left.z \sim z_{0}\right)$ where self-focusing appears to overcome plasma defocusing, leading to filamentation collapse, then the plasma density rises again, the filamentation recovers, and a dynamic balance is reestablished. From the observed filament contrast, however, in general, $\Delta n^{\text {lin }}>$ $\Delta n^{\text {circ }}$. The filamentation differences were less obvious as the inscription depth was increased beyond $4 \mathrm{~mm}$, but still apparent even at $6 \mathrm{~mm}$ depth.

\section{Discussion}

Filamentary femtosecond modification is accomplished with parallel beams with $\tau_{\mathrm{P}} \sim 180 \mathrm{fs}$ pulselength at $\lambda_{2 \omega}=387 \mathrm{~nm}$ and low NA (0.08). Filamentation, or self-guiding with intense femtosecond pulses, has been observed in air [34] and fused silica $[35,36]$, involving complex nonlinear processes such as self-focusing, multi-photon absorption, pulse steepening, and

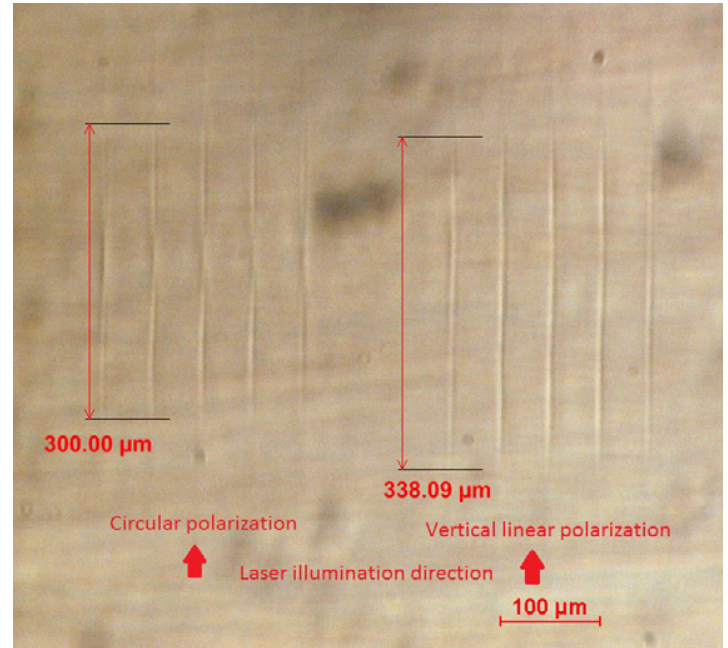

(a)

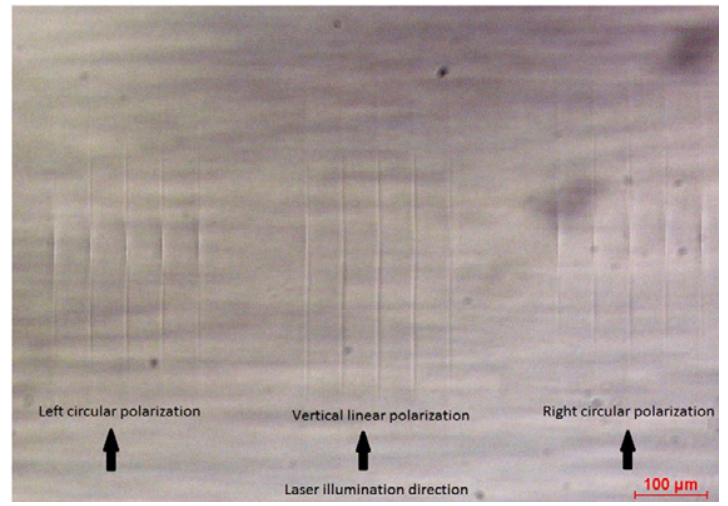

(b)

Figure 9. (a) Top: optical image of filaments $\sim 1$ day after inscription produced with circular $|R\rangle$ and linear (TE) polarizations inscribed $0.5 \mathrm{~mm}$ below surface with identical pulse energies. (b) Bottom: filaments inscribed $2 \mathrm{~mm}$ deep with $|R\rangle,|V\rangle$, and $|L\rangle$ polarizations, again showing higher contrast with linear polarization and similar inscription with both $|R\rangle$ and $|L\rangle$.

self-phase modulation, generally leading to a supercontinuum, depending on the material bandgap [37]. Self-guiding is the result of the dynamic balance between self-focusing (Kerr lensing) and plasma defocusing, while effects such as chromatic dispersion are expected to be much less important [38]. This leads to spatial confinement over distances much greater than the characteristic Rayleigh length. When a Gaussian laser pulse of peak intensity $I\left(\mathrm{~W} \mathrm{~cm}^{-2}\right)$ with plane wavefront passes through a transparent dielectric (Kerr) medium, the refractive index $n_{0}$ is modulated instantaneously to $n=n_{0}+n_{2} I$, where $n_{2}$ is the nonlinear RI component generated through the third-order susceptibility $\chi^{(3)}$. As the pulse propagates, the phase delay on the axis is greater than at the pulse edge; hence an instantaneous lens is formed, leading to self-focusing if the peak power exceeds the critical power [39],

$$
P_{\mathrm{c}}=\left[\frac{\pi(0.61)^{2} \lambda_{0}^{2}}{8 n_{0} n_{2}}\right],
$$


overcoming diffraction. Theoretically, self-focusing would lead to a singularity, but it is prevented from doing so by plasma defocusing. As the intensity increases, nonlinear MPI generates seed electrons in the conduction band, followed by impact ionization; hence a free electron plasma with density $\rho_{\mathrm{e}}(t)$ grows exponentially (highest density $\rho_{\mathrm{e}}$ on axis). This creates a negative lens which balances the self-focusing, leading to confinement of the radiation in a filament, where the ultrahigh intensity (here, $I \sim 1.2 \times 10^{13} \mathrm{~W} \mathrm{~cm} \mathrm{~cm}^{-2}$ ) is clamped [35]. The filament lengths observed here are $\delta L \sim 338 \mu \mathrm{m}$ (figure 9(a)) while the Rayleigh length $R_{\mathrm{L}}=$ $\left(\pi n_{0} \frac{\omega_{0}^{2}}{\lambda_{0}}\right)=48.4 \mu \mathrm{m}\left(\omega_{0}=2 \mu \mathrm{m}, \lambda_{0}=0.387 \mu \mathrm{m}\right)$; hence $\delta L \gg R_{\mathrm{L}}$, as expected. During the study of transient plasmas in fs waveguiding in doped phosphate glass with temporal pulselengths $100<\tau_{\mathrm{p}}<400 \mathrm{fs}$, Gawelda et al [40] observed clear polarization-dependent effects in filamentation which diminished with increasing temporal pulselength and fully suppressed at $400 \mathrm{fs}$ with circular polarization while still being present with linear polarization. The results were consistent with a difference in critical powers: $P_{\mathrm{C}}^{\text {lin }}=1.03 \mathrm{MW}$ and $P_{\mathrm{C}}^{\text {circ }}=1.53 \mathrm{MW}$, a factor of $\sim 1.5$ higher. The ratio of the nonlinear refractive indices $n_{2}^{\text {lin }}$ and $n_{2}^{\text {circ }}$ in an isotropic dielectric medium scale as $n_{2}^{\text {circ }} / n_{2}^{\text {lin }}=1.5$ [39] due to the fact that the third-order susceptibility $\chi^{(3)}$ (which is a tensor) has only one component with circular polarization whereas the linear polarization has two components. The ratio of critical powers is expected to be $P_{\mathrm{C}}^{\text {circ }} / P_{\mathrm{C}}^{\text {lin }}=1.5$; thus self-focusing is weaker with circular than linear polarization and consistent with observations during filamentation in a pure polymer, PMMA.

PMMA has a band gap of $E_{\mathrm{b}}=4.58 \mathrm{eV}$ [25], so that MPI is initiated by 2-photon absorption at $387 \mathrm{~nm}(h v=3.2 \mathrm{eV})$, and since the temporal pulselength is $\tau_{\mathrm{p}} \sim 180 \mathrm{fs}$ impact ionization is also significant. The electron density may reach $\rho_{\mathrm{e}}>10^{19} \mathrm{~cm}^{-3}$, where irreversible modification occurs. The peak intensity $I$ in each filament can be estimated to be $I=\left(\frac{2 E_{\mathrm{P}}}{\pi \omega_{0}^{2} \tau_{\mathrm{P}}}\right) \sim 1.22 \times 10^{13} \mathrm{~W} \mathrm{~cm}^{-2}$ with $1 / e^{2}$ focal spot size $2 \omega_{0} \sim \frac{1.22 \lambda}{\mathrm{NA}}=5.9 \mu \mathrm{m}$, while the peak power of each diffracted beam is $P=1.7 \mathrm{MW}\left(\frac{0.31 \mu \mathrm{J}}{180 \mathrm{fs}}\right)$.

The observed filamentation differences (figures 9(a), (b)) with linear and circular polarizations indicate that the ratio of critical powers $P_{\mathrm{C}}^{\text {lin }} / P_{\mathrm{C}}^{\text {circ }}>1$ in PMMA and that filamentation leads to stronger coupling and higher $\langle\Delta n\rangle$ with linear polarization. The relationship between $n_{2}$ and the third-order susceptibility $\chi^{(3)}$ is given by [41]

$$
n_{2}=\frac{0.0395 \chi^{(3)}}{n_{0}^{2}}
$$

where $n_{2}$ has units $\mathrm{cm}^{2} \mathrm{~W}^{-1}$ and $\chi^{(3)}$ is measured in esu. In optical solids, the value of $\chi^{(3)}$ is typically in the range $10^{-13}-10^{-14}$ esu [40]. For example, the value of $\chi^{(3)}$ in PMMA at $\lambda=1500 \mathrm{~nm}$ was estimated to be [42] $\chi^{(3)} \sim 3 \times 10^{-14}$ esu and, as $\chi^{(3)}$ scales linearly with wavelength, then $\chi_{387}^{(3)} / \chi_{1500}^{(3)}=0.258$, yielding $\chi_{387}^{(3)} \sim 7.7 \times$ $10^{-15}$; hence $n_{2}^{387} \sim 1.37 \times 10^{-16} \mathrm{~cm}^{2} \mathrm{~W}^{-1}$. The critical power for self-focusing in PMMA (equation (2)) is therefore
$P_{\mathrm{C}} \sim 1.1 \mathrm{MW}$, while the peak power in each beam $P=$ $1.7 \mathrm{MW} \sim 1.5 P_{\mathrm{C}}$, which confirms that self-focusing will occur ahead of the geometrical focus inside the material. The value of $\chi^{(3)}$ quoted by D'Amore et al [42] was measured on a thin film of PMMA by third-harmonic generation and with accuracy limited by significant background from the fused silica substrate. This places an uncertainty on the critical power calculated at $387 \mathrm{~nm}$ above; hence we have also estimated $n_{2}^{387}$ as follows, with a very simple model [39].

From the observed filament widths, self-guiding was limited to a diameter $\varphi=4.0 \mu \mathrm{m}$ during filamentation. This can be compared to a waveguide whose core has $\mathrm{RI}=n_{0}+\delta n$, while outside $\mathrm{RI}=n_{0}$. The critical angle for traversing rays in a waveguide is given by $\theta_{\mathrm{c}}=\left(2 \delta n / n_{0}\right)^{1 / 2}$, while the angle of diffraction of a Gaussian beam of diameter $\varphi$ is given by $\theta_{\text {dif }}=$ $0.61 \lambda / n_{0} \varphi$, so guiding will occur if all rays experience total internal reflection, that is when $\theta_{\mathrm{c}}=\left(2 \delta n / n_{0}\right)^{1 / 2}=\theta_{\text {dif }}=$ $0.61 \lambda / n_{0} \varphi$; hence

$$
\delta n_{387}=n_{2}^{387} I=n_{0} \frac{\left(0.61 \lambda_{0}\right)^{2}}{2\left(n_{0} \varphi\right)^{2}} .
$$

With $I=1.22 \times 10^{13} \mathrm{~W} \mathrm{~cm}{ }^{-2}, \lambda_{0}=3.87 \times 10^{-5} \mathrm{~cm}$, $\varphi=4.0 \times 10^{-4} \mathrm{~cm}$, and $n_{0}=1.49$, we obtain a value $n_{2}^{387}=9.58 \times 10^{-17} \mathrm{~cm}^{2} \mathrm{~W}^{-1}$, in reasonable agreement with the value in D'Amore [42] $\left(1.37 \times 10^{-16} \mathrm{~cm}^{2} \mathrm{~W}^{-1}\right)$ and lower by a factor of 0.7 . The proximity of these values supports the linear scaling of $\chi^{(3)}$ to shorter wavelengths. The RI modulation during self-guiding in PMMA is $\delta n=$ $n_{2}^{387} I \sim 1.67 \times 10^{-3}$, a level consistent with a strongly guiding structure. Interestingly, a collimated beam at $387 \mathrm{~nm}\left(2 \omega_{0}=\right.$ $0.8 \mathrm{~cm}$ ) with peak power $P \sim 1.7 P_{\mathrm{C}}$ traversing a $10 \mathrm{~cm}$ thick PMMA sample should self-focus in a distance [39] $Z_{\mathrm{sf}}=$ $\omega\left(n_{0} / 2 n_{2} I\right)^{0.5} \sim 8.4 \mathrm{~cm}$, and then avoid collapse through filamentation.

The plasma electron density, $\varrho_{\mathrm{e}}$, which balances the self-focusing attained in the filaments, can be estimated from the real part $R_{\mathrm{e}}(\Delta n)$ of the Drude equation for the RI change due to the plasma [36],

$$
\Delta n=n_{2}^{387} I=\left[\frac{e^{2}}{2 n_{0} \varepsilon_{0} \omega^{2} m_{\mathrm{e}}}\right]\left[\frac{\rho_{\mathrm{e}} \omega^{2} \tau_{\mathrm{c}}^{2}}{1+\omega^{2} \tau_{\mathrm{c}}^{2}}\right],
$$

where $e$ and $m_{\mathrm{e}}$ are the electron's charge and mass, $\varepsilon_{0}$ is the permittivity of free space, $n_{0}(1.49)$ is the linear refractive index, $\rho_{\mathrm{e}}$ is the electron density, $\omega(387 \mathrm{~nm})$ is the laser frequency $\left(3.87 \times 10^{15} \mathrm{rad} \mathrm{s}^{-1}\right)$, and $\tau_{\mathrm{c}}$ is the electron collision time. If $\tau_{\mathrm{c}}$ is of the order of $1 \mathrm{fs}$ or greater $\left(\tau_{\mathrm{c}}=0.4 \mathrm{fs}\right.$ in fused silica), the ratio $\omega^{2} \tau_{\mathrm{c}}^{2} /\left(1+\omega^{2} \tau_{\mathrm{c}}^{2}\right)>0.96$ and it tends closer to unity as $\tau_{\mathrm{c}}$ increases. Hence we obtain, as $n_{2} I=1.67 \times 10^{-3}$, an estimate of the electron density in a filament, with the help of equation (5):

$$
\begin{aligned}
\rho_{\mathrm{e}} & =\frac{2 n_{0} \varepsilon_{0} \omega^{2} m_{\mathrm{e}}\left(n_{2} I\right)}{e^{2}} \\
& =2.22 \times 10^{22} \cdot\left(n_{2} I\right)=4.3 \times 10^{19} \mathrm{~cm}^{-3} .
\end{aligned}
$$

This value is of the correct order required to produce permanent modification [37] and well below the critical plasma density $\rho_{\mathrm{e}}^{\text {crit }} \sim 10^{21} \mathrm{~cm}^{-3}$ which would result in 
complete optical breakdown. As the scan speed $s=1 \mathrm{~mm} \mathrm{~s}^{-1}$ during inscription, corresponding to $s=1 \mu \mathrm{m} \mathrm{ms}^{-1}$, the material is exposed to only $\sim 4$ overlapped pulses during inscription of $4 \mu \mathrm{m}$ wide structures.

As $n_{2}^{\text {lin }}>n_{2}^{\text {circ }}$, equation (6) infers that the plasma density reached with linear polarization should be higher than that with circular polarization. Diffraction efficiencies measured soon after inscription should therefore reflect the degree of bond breaking in the polymer, dependent on the plasma formation during filamentation.

The grating efficiencies depend on the phase angle $\phi$, where $\phi(|\mathrm{P}\rangle)=\pi \Delta n_{\mathrm{P}} L / \lambda \cos \theta_{\mathrm{B}}$ for a grating of length $L$ and $|\mathrm{P}\rangle$ is the state of polarization. As the inscribed filament cross sections for linear and circular polarizations are different, then allowing for slight variation in $\Delta n_{\mathrm{P}}$ with depth $z$, the difference in first-order DEs is related to the changes in optical path length OPD $=\delta\left(\Delta n_{\mathrm{P}} L\right)$, given by

$$
\begin{aligned}
\delta(\mathrm{OPD}) & =\int\left[\Delta n^{\operatorname{lin}}(z)-\Delta n^{\mathrm{circ}}(z)\right] \mathrm{d} z \\
& =\left[\left\langle\Delta n^{\operatorname{lin}}\right\rangle-\left\langle\Delta n^{\text {circ }}\right\rangle\right] L .
\end{aligned}
$$

The observed DE after 1 day for linear (TE) polarization was $62 \%$ (figure 5), while the ratio of diffraction efficiencies $R=\left(\sin ^{2}\left\langle\alpha \Delta n^{\operatorname{lin}}\right\rangle / \sin ^{2}\left\langle\alpha \Delta n^{\text {circ }}\right\rangle\right)=1.21 \pm 0.03$ after $\sim 1$ day, which leads to $\left(\left\langle\Delta n^{\operatorname{lin}}\right\rangle /\left\langle\Delta n^{\text {circ }}\right\rangle\right)_{\max } \sim 1.13 \pm 0.03$ when averaged throughout a $4 \mathrm{~mm}$ grating structure, a modest though significant variation.

As 2-photon absorption (also related to third-order susceptibility $\chi^{(3)}$ ) initiates the nonlinear ionization process in PMMA at $387 \mathrm{~nm}$, there may be a polarization-dependent effect on the 2-photon absorption cross section ratios in PMMA which initiates plasma formation.

Effects of polarization in multi-photon ionization (MPI) were first observed in 1971 during the 3-photon ionization of atomic caesium with a ruby laser where experimentally, the ratio of cross sections was found to be $\left(\frac{\sigma_{3}^{\text {circ }}}{\sigma_{3}^{\operatorname{lin}}}\right)=2.15 \pm$ 0.4 [43]. Following this, experiments on 2-photon ionization of caesium yielded the ratio $\left(\frac{\sigma_{2}^{\text {circ }}}{\sigma_{2}^{\operatorname{lin}}}\right)=1.28 \pm 0.2$ [44]. These experiments inspired theoretical work on MPI [45, 46] which predicted the dominance of circular polarization in low-order MPI, $N \leq 3$ with upper limits $\left(\frac{\sigma_{2}^{\text {circ }}}{\sigma_{2}^{\text {lin }}}\right)=3 / 2$ and $\left(\frac{\sigma_{3}^{\text {circ }}}{\sigma_{3}^{\operatorname{lin}}}\right)=5 / 2$, so the theory and experiments were in reasonable agreement. The general arguments related to angular momentum conservation during MPI were expected to apply also in solids, confirmed with 4-photon conductivity experiments in quartz, yielding $\left(\frac{\sigma_{2}^{\text {circ }}}{\sigma_{2}^{\text {lin }}}\right)=2.05 \pm 0.9$ [47]. Reiss [46] pointed out, however, that a significant reversal of this behaviour was expected for $N>4$ and confirmed in high $N$, 6-photon resonance-enhanced MPI (REMPI, $3+3$ ), in the NO molecule, yielding $\left(\frac{\sigma_{6}^{\text {lin }}}{\sigma_{6}^{\text {circ }}}\right) \sim 2.9$ [48]. In addition, single-pulse, (50 fs, $800 \mathrm{~nm}$ ) 6-photon ionization in fused silica at peak intensities $I \sim 10-20 \mathrm{TW} \mathrm{cm}^{-2}$ yielded the ratio of absorption cross sections $\left(\frac{\sigma_{6}^{\text {lin }}}{\sigma_{6}^{\text {circ }}}\right) \sim 3.7$ [49]. However, the RI contrast during waveguide inscription in fused silica (120 fs, $1 \mathrm{kHz}, 800 \mathrm{~nm}, 0.46 \mathrm{NA}$ ) was both polarization and intensity dependent [50].

The plasma densities reached in filaments (equation (6)) with linear and circular polarization could be expected to vary by a factor of $3 / 2$, leading to $\left(\left\langle\Delta n^{\operatorname{lin}}\right\rangle /\left\langle\Delta n^{\text {circ }}\right\rangle\right)_{\text {theory }} \sim$ 1.5 , assuming that $\Delta n \ltimes \rho_{\mathrm{e}}$. However, the ratio is clearly much lower, $\left(\left\langle\Delta n^{\operatorname{lin}}\right\rangle /\left\langle\Delta n^{\text {circ }}\right\rangle\right) \sim 1.13$. However, if the ratio of two-photon ionization cross sections in PMMA was similar that observed in atomic caesium, that is, favouring circular polarization, with $\left(\frac{\sigma_{2}^{\text {circ }}}{\sigma_{2}^{\operatorname{lin}}}\right) \sim$ 1.28 [44], then circular polarization could enhance 2-photon ionization over linear polarization, increasing the initial seed electron density available for impact ionization. This effect could reduce the differential in final plasma densities, yielding $\left(\left\langle\Delta n^{\text {lin }}\right\rangle /\left\langle\Delta n^{\text {circ }}\right\rangle\right) \sim \frac{1.5}{1.28}=1.17$, closer to the ratio $\left(\left\langle\Delta n^{\text {lin }}\right\rangle /\left\langle\Delta n^{\text {circ }}\right\rangle\right)_{\max } \sim 1.13 \pm 0.03$ estimated from the measured first-order diffraction efficiencies. This may be a fortuitous result and would require additional confirmation by high NA, polarization-dependent experiments (defeating filamentation) of 2-photon absorption coefficients, well beyond the scope of the current paper.

The photochemical changes in pure PMMA after fs inscription with NUV pulses at $387 \mathrm{~nm}$ have previously been analysed in detail [25] and, more recently, Raman mapping after fs exposure at $400 \mathrm{~nm}$ confirmed the increased density (relative to surrounding material) of $\mathrm{C}=\mathrm{C}$ bonds, detected at wavenumber $\varpi=1640 \mathrm{~cm}^{-1}$ [51]. These bonds are found only in the monomer and end groups of PMMA, supporting the view that fs exposure in PMMA causes chain scission of the polymer backbone and depolymerization [25]. The temporal development over 30 days might well be due to diffusion of monomer MMA into unexposed regions combined with cross linking and stress relaxation. As linear polarization appears to couple strongest to the substrate, we infer that it creates more chain scission (bond breaking) in filaments than with circular polarization [52]. The $\Delta n \mathrm{~s}$ observed after exposure, and differences due to polarization, although modest, support this view.

\section{Conclusions}

We have used low NA, parallel beam NUV fs inscription with a phase-only SLM to create high efficiency VBGs in pure PMMA with thickness $L=1-7 \mathrm{~mm}$, reaching the first-order Bragg efficiency $\eta_{1} \sim 94 \%$ with $L=4 \mathrm{~mm}$, when inscribed with linear vertical polarization (TE) perpendicular to the scan direction. The diffraction efficiency is the highest achieved in a pure polymer as far as the authors are aware. An excellent fit to Kogelnik theory $\eta_{ \pm 1}=\sin ^{2} \varphi$ was observed [31] and used to estimate the RI modulation, which reached $\Delta n_{\mathrm{TE}}^{\operatorname{lin}}=(6.01 \pm 0.07) \times 10^{-5}$ after 30 days. The excellent fit to the theoretical curve infers that VBGs are approaching device level quality. By inscribing sets of $5 \mathrm{~mm}$ thick VBGs with the same pulse energy but different linear and circular polarizations $|V\rangle,|H\rangle,|R\rangle,|L\rangle$, 
maximum diffraction efficiency was observed with linear vertical polarization $|V\rangle$ (TE), then horizontal $|H\rangle$ (TM) (TM), and lowest with circular polarization. The ratio of first-order DEs was $\left[\eta_{1}(|V\rangle) / \eta_{1}(|R\rangle)\right]_{\max }=1.21 \pm 0.03$ soon after inscription. By studying the filamentary inscription cross sections made under identical energies but with linear (TE) and circular polarizations, a polarization-dependent filamentary modification was confirmed, stronger with linear than circular polarization. This is consistent with the observed stronger self-focusing with linear than circular polarization, and was theoretically predicted [39]. The observed ratio of diffraction efficiencies infers that $\left(\left\langle\Delta n^{\operatorname{lin}}\right\rangle /\left\langle\Delta n^{\text {circ }}\right\rangle\right)_{\max }=$ $1.13 \pm 0.03$ when averaged over a $5 \mathrm{~mm}$ thick structure.

While linear polarization gave highest $\Delta n$, inscribed VBGs displayed higher background (6-8\%) than with circular polarization, 2-3\%. The source of the background is under investigation. There may, for example, be a remaining small stitching error or possibly an effect due to imperfect uniformity between the parallel spots. In the case of linear (TE) polarization, periodic nanostructures can be written within the filaments during exposure, increasing coupling to the plasma due to the interference of the laser electric field with bulk electron plasma waves [53]. Surface ablation of PMMA with $45 \mathrm{fs}, 800 \mathrm{~nm}$ pulses shows a strong asymmetry due to polarization dependence [54]. The inscription symmetry offered by circular polarization is clearly desirable to avoid such effects.

\section{Acknowledgments}

We are grateful to Professor Miles Padgett of Glasgow University for the use of the Labview software for calculating phase CGHs.

\section{References}

[1] Arns J A, Colburn W C and Barden S C 1999 Proc. SPIE 3779 313-23

[2] Volodin B L, Dolgy S V, Melnik E D, Shaw J and Ban V S 2004 Opt. Lett. 29 1891-3

[3] Glebov A L, Mokhun O, Rapaport A, Vergnole S, Smirnov V and Glebov L B 2012 Proc. SPIE 8428 84280C

[4] Liao K H et al 2007 Opt. Express 15 4876-82

[5] Shankoff T A 1968 Appl. Opt. 7 2101-5

[6] Efimov O M, Glebov L B, Glebova L N, Ricahrdson K C and Smirnov V I 1999 Appl. Opt. 38 619-27

[7] Ciapurin I G, Glebov L B, Glebova L N, Smirnov V L and Rotari E V 2003 Proc. SPIE 4974 209-19

[8] Davis K M, Miura K, Sugimoto N and Hirao K 1996 Opt. Lett. 21 1729-31

[9] Ossellame R, Cerulloa G and Ramponi R (ed) 2011 Fs Laser Micro-Machining (Topics in Applied Physics vol 123) (Heidelberg: Springer) pp 155-95

[10] Watanabe W and Itoh K 2004 Proc. SPIE 5340 119-26

[11] Paipulas D, Kudriasov V, Kurselis K, Malinauskas M, Ost S and Sirutkaitis V 2010 LPM 2010: Proc. 11th Int. Symp. on Laser Precision Microfabrication (Stuttgart, June 2010)

[12] Voiglander C, Richter D, Thomas J, Tunnermann A and Nolte S 2010 Appl. Phys. A 102 35-8

[13] Lee D, Thomson R R and Cunningham C R 2012 Instrumentation and methods for astrophysics arXiv:1207.2661 [astro-phIM]
[14] Yamasaki K, Juodkazis S, Lippert T, Watanabe M, Matsuo S and Misawa H 2003 Appl. Phys. A 76 325-9

[15] Mizeikes V, Seet K K, Juodkasis S and Misawa H 2004 Proc. SPIE 5662 95-100

[16] Zoubir A, Lopez C, Richardson M and Richardson K 2004 Opt. Lett. 29 1840-2

[17] Watanabe W, Sowa S, Tamaki T, Itoh K and Nishii J 2006 Japan. J. Appl. Phys. 45 L765-7

[18] Yamasaki K, Juodkazis S, Matsuo S and Misawa H 2003 Appl. Phys. A 77 371-3

[19] Uppal N, Shiakolas P S and Rizwan M 2008 Proc. SPIE $\mathbf{6 8 8 2} 68820 \mathrm{I}$

[20] Si J, Qiu J, Zhai J, Shen Y and Hirao K 2002 Appl. Phys. Lett. 80 359-61

[21] Scully P J, Jones D and Jaroszynski D A 2003 J. Opt. A: Pure Appl. Opt. 5 S92-6

[22] Mochizuki H, Watanabe W, Ezoe R, Tamaki T, Ozeki Y, Itoh K, Kasuya M, Matsuda K and Hirono S 2008 Appl. Phys. Lett. 92091120

[23] Baum A, Scully P J, Perrie W, Jones D, Isaac R and Jaroszynski D A 2008 Opt. Lett. 33 651-3

[24] Watanabe W 2009 Laser Phys. 19 342-5

[25] Baum A, Scully P J, Basanta M, Paul Thomas C L, Fielden P R, Goddard N J, Perrie W and Chalker P 2007 Opt. Lett. 32 190-2

[26] Hasegawa S and Hayasaki Y 2007 Proc. SPIE 6458 1-15

[27] Liu D, Kuang Z, Perrie W, Scully P J, Baum A, Edwardson S P, Fearon E, Dearden G and Watkins K G 2010 Appl. Phys. B 101 817-23

[28] Liu D, Perrie W, Kuang Z, Scully P J, Baum A, Liang S, Edwardson S P, Fearon E, Dearden G and Watkins K G 2012 Appl. Phys. B 107 795-801

[29] Leach J, Sinclair G, Jordan P, Courtial J, Padgett M J and Laczik Z 2004 Opt. Express 12220

[30] Kazansky P G, Yang W, Briochi E, Bovatsek J, Arai A, Shimotsuma Y, Miura K and Hirao K 2007 Appl. Phys. Lett. 90151120

[31] Kogelnik H 1969 Bell Syst. Tech. J. 482909

[32] Hnatovski C, Taylor R S, Simova E, Bhardwai V R, Raynor D M and Corkum P B 2005 J. Appl. Phys. 98013517

[33] Ciapurin I V, Glebov L B and Smirnov V I 2005 Proc. SPIE 5742183

[34] Braun A, Korn G, Liu X, Du D, Squier J and Mourou G 1995 Opt. Lett. 2073

[35] Tzortzakis S, Sudrie L, Franco M, Prade B, Mysyrowicz A, Couairon A and Berge L 2001 Phys. Rev. Lett. 87213902

[36] Tzortzakis S, Papazoglou D G and Zergioti I 2006 Opt. Lett. 31 796-8

[37] Brodeur A and Chin S L 1998 Phys. Rev. Lett. 80 4406-9

[38] Couairon A, Gaizauskas E, Faccio D, Dubietis A and Di Trapani P 2006 Phys. Rev. E 73016608

[39] Boyd R W 2008 Nonlinear Optics 3rd edn (Burlington, MA: Academic) chapter 4, pp 207-52

[40] Gawelda W, Puerto D, Seigal J, Ferrer A, Ruiz de la Cruz A, Fernadez H and Solis J 2008 Appl. Phys. Lett. 93121109

[41] Boyd R W and Fischer G L 2001 Nonlinear optical materials Encyclopedia of Materials; Science and Technology (Oxford: Elsevier) pp 6237-44

[42] D'Amore F, Lanata M, Pietralunga S M, Gallazzi M C and Zerbi G 2004 Opt. Mater. 24 661-5

[43] Fox R A, Kogan R M and Robinson E J 1971 Phys. Rev. Lett. 261416 
[44] Kogan R M, Fox R A, Burnham G T and Robinson E J 1971 Bull. Am. Phys. Soc. 161411

[45] Klarsfeld S and Maquet A 1972 Phys. Rev. Lett. 29 79-81

[46] Reiss H R 1972 Phys. Rev. Lett. 29 1129-31

[47] Venable D D and Kay R B 1975 Appl. Phys. Lett. 2748

[48] Carman H S and Compton R N 1989 J. Chem. Phys. 901307

[49] Temnov V V, Sokolowsli-Tinten K, Zhou P, El-Khamhawy A and von der Linde D 2006 Phys. Rev. Lett. 97237403

[50] Little D J, Ams M, Dekker P, Marshall G D, Dawes J M and Withford M J 2008 Opt. Express 1620029
[51] Pang B, Taranu A, Liang S, Casabella S, Fiadzomor P and Scully P 2011 POF 2011: Proc. 20th Int. Conf. Plastic Optical Fibers (Bilbao, Sept. 2011)

[52] Baum A, Scully P J, Perrie W, Liu D and Lucarini V 2010 J. Opt. Soc. Am. B 27 107-11

[53] Shimotsuma Y, Kazansky P G, Qiu J and Hirao K 2003 Phys. Rev. Lett. 91247405

[54] Guay J M, Villafranca A, Baset F, Popov K, Ramunno L and Bhardwaj V R 2012 New J. Phys. 14085010 